\title{
Optimum levels of exchangeable protons in perdeuterated proteins for proton detection in MAS solid-state NMR spectroscopy
}

\author{
Ümit Akbey $\cdot$ Sascha Lange $\cdot$ W. Trent Franks · \\ Rasmus Linser · Kristina Rehbein · Anne Diehl · \\ Barth-Jan van Rossum · Bernd Reif · Hartmut Oschkinat
}

Received: 18 June 2009/ Accepted: 23 July 2009/Published online: 22 August 2009

(C) Springer Science+Business Media B.V. 2009

\begin{abstract}
We present a systematic study of the effect of the level of exchangeable protons on the observed amide proton linewidth obtained in perdeuterated proteins. Decreasing the amount of $\mathrm{D}_{2} \mathrm{O}$ employed in the crystallization buffer from 90 to $0 \%$, we observe a fourfold increase in linewidth for both ${ }^{1} \mathrm{H}$ and ${ }^{15} \mathrm{~N}$ resonances. At the same time, we find a gradual increase in the signal-to-noise ratio (SNR) for ${ }^{1} \mathrm{H}-{ }^{15} \mathrm{~N}$ correlations in dipolar coupling based experiments for $\mathrm{H}_{2} \mathrm{O}$ concentrations of up to $40 \%$. Beyond $40 \%$, a significant reduction in SNR is observed. Scalarcoupling based ${ }^{1} \mathrm{H}-{ }^{15} \mathrm{~N}$ correlation experiments yield a nearly constant SNR for samples prepared with $\leq 30 \% \mathrm{H}_{2} \mathrm{O}$. Samples in which more $\mathrm{H}_{2} \mathrm{O}$ is employed for crystallization show a significantly reduced NMR intensity. Calculation of the SNR by taking into account the reduction in ${ }^{1} \mathrm{H} T_{1}$ in samples containing more protons (SNR per unit time), yields a maximum SNR for samples crystallized using 30 and $40 \%$ $\mathrm{H}_{2} \mathrm{O}$ for scalar and dipolar coupling based experiments, respectively. A sensitivity gain of 3.8 is obtained by increasing the $\mathrm{H}_{2} \mathrm{O}$ concentration from 10 to $40 \%$ in the $\mathrm{CP}$ based experiment, whereas the linewidth only becomes 1.5 times broader. In general, we find that $\mathrm{CP}$ is more favorable compared to INEPT based transfer when the number of possible ${ }^{1} \mathrm{H},{ }^{1} \mathrm{H}$ interactions increases. At low levels of deuteration $\left(\geq 60 \% \mathrm{H}_{2} \mathrm{O}\right.$ in the crystallization buffer), resonances from rigid residues are broadened beyond detection. All experiments are carried out at MAS frequency of
\end{abstract}

Ümit Akbey and Sascha Lange contributed equally.

Ü. Akbey · S. Lange · W. Trent Franks · R. Linser ·

K. Rehbein · A. Diehl · B.-J. van Rossum · B. Reif .

H. Oschkinat $(\bowtie)$

Leibniz-Forschungsinstitut für Molekulare Pharmakologie

(FMP), Robert-Rössle-Str. 10, 13125 Berlin, Germany

e-mail: oschkinat@fmp-berlin.de
$24 \mathrm{kHz}$ employing perdeuterated samples of the chicken $\alpha$-spectrin SH3 domain.

Keywords Proton detection - Deuteration - Protein · MAS NMR $\cdot \alpha$-spectrin SH3

\section{Introduction}

Proton detection has been utilized successfully for the $\alpha$-spectrin SH3 domain (Chevelkov et al. 2003; Linser et al. 2008) and GB1 (Zhou et al. 2007) to obtain high-resolution spectra of biological samples. A theoretical increase in sensitivity of $\sim 31$ (sensitivity gain $\approx\left(\gamma_{H} / \gamma_{N}\right)^{3 / 2}$ ) is expected for ${ }^{1} \mathrm{H}$ detection as compared to ${ }^{15} \mathrm{~N}$ detection (Ishii and Tycko 2000). The value of enhancement in resolution and sensitivity depends crucially on the experimental linewidths of proton and nitrogen signals (Chevelkov et al. 2003). To improve the resolution of proton detected solid-state NMR experiments, fast magic-angle spinning (MAS) (Paulson et al. 2003; Zhou et al. 2006; Laage et al. 2009), application of homonuclear decoupling sequences (Vinogradov et al. 1999; Rossum et al. 2003; Salager et al. 2009), and reduction of the proton homonuclear coupling network by extensive deuteration have been proposed (Chevelkov et al. 2006).

Application of fast-MAS implicates the use of small diameter rotors. Recently, experiments on fully protonated GB1 protein, spinning at a frequency of $40 \mathrm{kHz}$ in a $1.6 \mathrm{~mm}$ rotor, resulted in proton linewidths of $\sim 360$ and $\sim 500 \mathrm{~Hz}$, at 750 and $500 \mathrm{MHz}$ proton frequency, respectively (Zhou et al. 2007). An increase in sensitivity of $\sim 14$ was observed when protons are detected instead of nitrogens in two-dimensional correlation experiments for GB1. In general, the reduced volume in small-diameter 
rotors puts a restriction to the absolutely achievable signal intensity. Homonuclear decoupling sequences provide a compromised sensitivity when applied in the direct dimension and will not be discussed here (Leskes et al. 2007). A significant reduction of the proton linewidth can be achieved with the help of suitable labeling schemes involving large scale deuteration of the protein (Chevelkov et al. 2006). A protonation level of $10 \%$ at exchangeable sites in an otherwise perdeuterated environment resulted in an effective linewidth on the order of 17-35 Hz (Chevelkov et al. 2006). A detailed comparison of the mentioned methods, MAS, homonuclear decoupling (FSLG) and deuteration has been performed by Zilm and his coworkers (Morcombe et al. 2005). It has been shown for ubiquitine that the best resolution was obtained for deuterated proteins, performing the experiments at a MAS frequency of $20 \mathrm{kHz}$ and using low-power heteronuclear decoupling. Extensive deuteration results in significant decrease of the Boltzman magnetization, and additionally in an increase in the proton $T_{1}$ relaxation time. Nevertheless, these drawbacks in sensitivity are compensated by the use of proton detection. By taking into account the reduction in proton and nitrogen linewidths, it has been shown that $10 \% \mathrm{H}_{2} \mathrm{O}$ $\mathrm{SH} 3$ has similar SNR compared to the $100 \% \mathrm{H}_{2} \mathrm{O} \mathrm{SH} 3$ (Chevelkov et al. 2006).

The goal of this investigation is to find the optimum concentration of $\mathrm{H}_{2} \mathrm{O}$ for the crystallization of a perdeuterated protein that still yields (i) an acceptable resolution, and (ii) maximizes sensitivity. In addition, we compare the magnetization transfer efficiency in dipolar (cross-polarization, CP) and scalar (INEPT) coupling based experiments varying the amount of $\mathrm{D}_{2} \mathrm{O}$ employed in the crystallization buffer from 90 to $0 \%$. The efficiency of a magnetization transfer is an important factor in pulse sequence design. In particular, implementation of more complex multidimensional experiments might require a careful analysis of the use of dipolar and scalar transfer sequences, especially when proton decoupling is considered.

\section{Experimental}

The SH3 domain of chicken $\alpha$-spectrin was utilized as a model system for the MAS NMR experiments presented in this study. The plasmid pET3d was used to code the $\alpha$-spectrin SH3 domain (P07751|965-1025) from chicken brain. The SH3 domain was expressed in E. coli BL21-DE3 grown on $100 \% \mathrm{D}_{2} \mathrm{O}$ based $\mathrm{M} 9$-minimal medium supplemented with $3 \mathrm{~g} / \mathrm{L} \mathrm{d}$ d $^{-}{ }^{13} \mathrm{C}$-Glucose, $1 \mathrm{~g} / \mathrm{L}{ }^{15} \mathrm{~N}-\mathrm{NH}_{4} \mathrm{Cl}$ (all isotopes were obtained from EURISO-TOP, Germany) and $60 \mathrm{mg} / \mathrm{L}$ carbenicilline. Cells from $0.2 \mathrm{~L} \mathrm{H}_{2} \mathrm{O}$ based M9 overnight culture were collected for inoculation of $1 \mathrm{~L}$ main $\mathrm{D}_{2} \mathrm{O}$ culture. Consequently, the growth started in contrast to the water based cultures at a relatively high $\mathrm{OD}_{600 \mathrm{~nm}}$ of 0.25 . Cells grew up to an $\mathrm{OD}_{600 \mathrm{~nm}}$ of 0.7 at $37^{\circ} \mathrm{C}$. The temperature was then decreased to $20^{\circ} \mathrm{C}$. Cells were induced with $1 \mathrm{mM}$ IPTG overnight. After purification, $7 \times 30 \mathrm{mg}$ of protein (in $\mathrm{H}_{2} \mathrm{O}, \mathrm{pH} 3.5$ with $0.02 \%$ Na-azide) was lyophilized and redissolved in water containing $90,80,70,60,40,20$ and $0 \% \mathrm{D}_{2} \mathrm{O}$ to yield a final protein concentration of $10 \mathrm{mg} \mathrm{SH} 3 / \mathrm{mL}$. The $\mathrm{pH}$ of all samples was then shifted to $\mathrm{pH} 7.5-8.5$ in a $\mathrm{NH}_{3}$ atmosphere. Samples were stored at $4^{\circ} \mathrm{C}$ for at least 3 days to allow for an optimum H/D exchange. To induce crystallization, an equal volume of a $200 \mathrm{mM} \mathrm{NH}_{4}\left(\mathrm{SO}_{4}\right)_{2}$ solution was added using water containing 90, 80, 70, 60, 40, 20 and $0 \% \mathrm{D}_{2} \mathrm{O}$, respectively. The $\mathrm{pH}$ was checked and tuned to 7.5 \pm 0.5 , if required. Microcrystals were allowed to settle for at least 3 days. Each $3.2 \mathrm{~mm}$ MAS rotor was filled with $\sim 15-20 \mathrm{mg}$ protein. The assignment of the spectra was reported previously (Rossum et al. 2003).

The details of the cross-polarization and INEPT type of polarization transfer schemes to record proton detected 2D ${ }^{1} \mathrm{H}-{ }^{15} \mathrm{~N}$ correlation spectra are described elsewhere (Paulson et al. 2003). The NMR experiments were performed on a Bruker Avance II spectrometer operating at $400 \mathrm{MHz}{ }^{1} \mathrm{H}$ Larmor frequency using a $3.2 \mathrm{~mm}$ triple resonance probe (Bruker, Karlsruhe, Germany). A $3.2 \mathrm{~mm}$ rotor size was chosen as a compromise between sample volume and MAS spinning frequency. The temperature of the samples was adjusted to $273 \mathrm{~K}$ in all experiments. The effective temperature was on the order of $\sim 22^{\circ} \mathrm{C}$ (Linser et al. 2008). The increase is due to frictional heating caused by fast sample rotation. A CP contact-time of $1.6 \mathrm{~ms}$ was employed for both $\mathrm{CP}$ steps in the $2 \mathrm{D}$ correlation experiments. The INEPT transfer time was kept at $\sim 2.17 \mathrm{~ms}$ in all experiments. A $7 \mathrm{~s}$ recycle delay was used for all experiments to prevent saturation effects (the longest bulk ${ }^{1} \mathrm{H} T_{1}, 4.28 \mathrm{~s}$, was observed for the $10 \%$ protonation level, see Table 1). $256 t_{1}$ experiments with 16 scans per increment were acquired, resulting in a total measurement time of $\sim 8 \mathrm{~h}$ for each recorded 2D CP and INEPT based experiment.

\section{Results and discussion}

One-dimensional (1D) ${ }^{15} \mathrm{~N}$ filtered ${ }^{1} \mathrm{H}$ MAS NMR spectra of the $\alpha$-spectrin SH3 protein prepared with different concentrations of protons at exchangeable sites are compared in Fig. 1. Obviously, the amide proton linewidths strongly depend on the proton density. All amide proton linewidths are extracted from 2D correlation spectra. The averaged values obtained for the different samples, are listed in Table 1.

For samples with 10 and $100 \%$ protons at exchangeable sites, average proton linewidths of $\sim 19$ and $\sim 58 \mathrm{~Hz}$ were 
Table 1 Average ${ }^{1} \mathrm{H}$ and ${ }^{15} \mathrm{~N}$ linewidths, ${ }^{1} \mathrm{H}$ and ${ }^{15} \mathrm{~N} T_{1}$ and $T_{2}$ bulk relaxation times, and average signal-to-noise ratios (SNR) for $\alpha$-spectrin $\mathrm{SH} 3$ obtained at a MAS rotation frequency of $24 \mathrm{kHz}$

\begin{tabular}{|c|c|c|c|c|c|c|c|}
\hline${ }^{1} \mathrm{H} \%$ & Average $^{\text {a }}{ }^{1} \mathrm{H}$ FWHM $(\mathrm{Hz})$ & Average ${ }^{15} \mathrm{~N}$ FWHM (Hz) & ${ }^{1} \mathrm{H} T_{1}(\mathrm{~s})$ & ${ }^{1} \mathrm{H} T_{2}(\mathrm{~ms})$ & ${ }^{15} \mathrm{~N} T_{2}(\mathrm{~ms})$ & Average SNR (CP) & Average SNR (INEPT) \\
\hline 10 & $19.0 \pm 3.3^{\mathrm{b}}$ & $11.3 \pm 5.4$ & 4.28 & 60.6 & 75.8 & $18.9 \pm 4.7$ & $50.6 \pm 16.6$ \\
\hline 20 & $20.2 \pm 5.7$ & $12.4 \pm 7.2$ & 2.76 & 55.3 & 57.3 & $33.5 \pm 11.4$ & $47.3 \pm 19.1$ \\
\hline 30 & $22.5 \pm 6.5$ & $15.4 \pm 7.5$ & 2.13 & 51.4 & 38.4 & $41.1 \pm 16.4$ & $50.4 \pm 25.2$ \\
\hline 40 & $27.5 \pm 6.1$ & $16.9 \pm 7.3$ & 1.67 & 33.1 & 37.5 & $44.6 \pm 22.6$ & $37.6 \pm 24.2$ \\
\hline 60 & $32.5 \pm 10.3$ & $25.1 \pm 13.5$ & 0.85 & 29.8 & 23.6 & $26.4 \pm 15.7$ & - \\
\hline 80 & $37.5 \pm 9.9$ & $32.3 \pm 14.3$ & 0.76 & 23.2 & 13.6 & $18.3 \pm 13.9$ & - \\
\hline 100 & $58.3 \pm 20.5$ & $41.2 \pm 14.4$ & 0.68 & 11.4 & 9.6 & $11.1 \pm 6.3$ & - \\
\hline
\end{tabular}

a At high concentrations of $\mathrm{H}_{2} \mathrm{O}(>60 \%)$, the FWHM is determined from the 'remaining' (detectable) resonances. The indicated value does not take into account resonances that are broadened beyond detection, i.e., the reported FWHM represents the lower limit of the actual line width

b The indicated values represent the deviation from the average FWHM

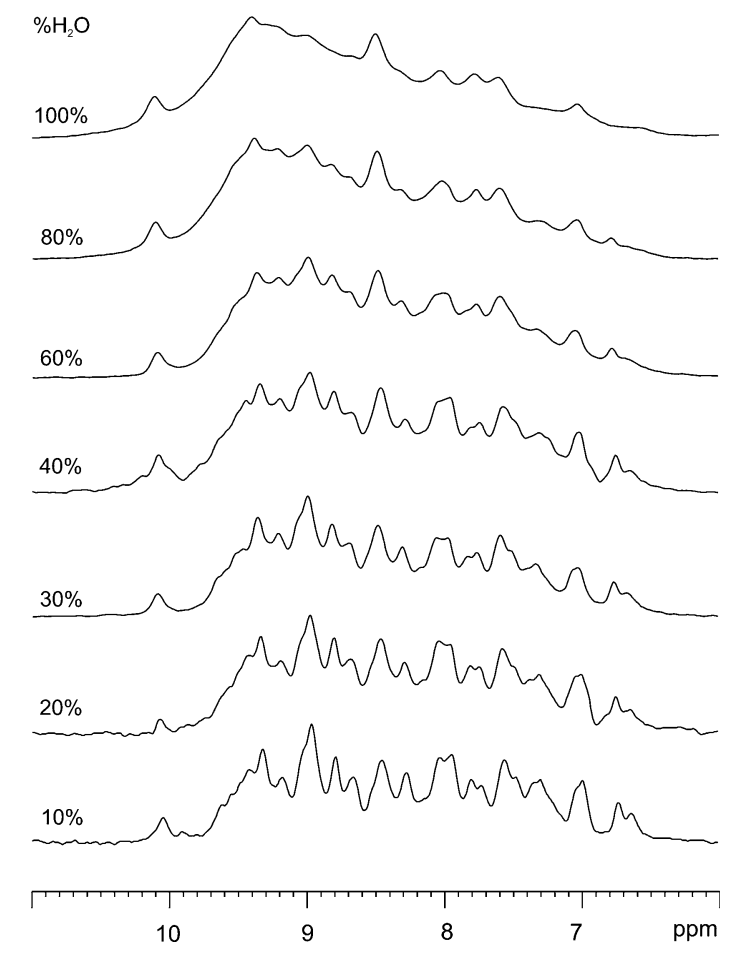

Fig. $1{ }^{15} \mathrm{~N}$-filtered ${ }^{1} \mathrm{H}$ 1D MAS NMR spectra of perdeuterated $\alpha$-spectrin SH3 samples precipitated with buffer containing $10-100 \%$ $\mathrm{H}_{2} \mathrm{O}$ and $90-0 \% \mathrm{D}_{2} \mathrm{O}$, respectively (bottom to top). The spectra were recorded at a MAS frequency of $24 \mathrm{kHz}$ and at $275 \mathrm{~K}$

obtained, respectively. The extent of resolution achieved with perdeuteration can be appreciated from a comparison of this proton line width to the line width obtained for protonated samples. In a fully protonated protein, the average proton linewidths is on the order of $\sim 300-450 \mathrm{~Hz}$ when the experiment is set up with heteronuclear detection and ${ }^{1} \mathrm{H},{ }^{1} \mathrm{H}$ homonuclear decoupling in the indirect ${ }^{1} \mathrm{H}$ dimension (Chevelkov et al. 2003).

The sensitivity enhancement factor, $\xi$, obtained by detection of ${ }^{1} \mathrm{H}$ instead of ${ }^{15} \mathrm{~N}$ can be described more quantitatively using the equation (Ishii et al. 2001), $\xi \approx\left(\frac{\gamma_{H}}{\gamma_{N}}\right)^{3 / 2}\left(\frac{W_{N}}{W_{H}}\right)^{1 / 2}\left(\frac{Q_{H}}{Q_{N}}\right)^{1 / 2} f$

$\gamma$ refers to the gyromagnetic ratio, $W$ represents the effective linewidth, $Q$ is the quality factor of the RF coil, and $f$ the efficiency of the polarization transfer between proton and nitrogen. The linewidth, $W$, is effected mostly by the amount of dipolar coupling present in the system, and can be tuned by the degree of deuteration.

The average signal-to-noise ratios (SNR) for the different samples are represented in Fig. 2a. We find that the optimum proton density at the exchangeable sites for the SH3 domain is obtained by using a ratio of 40:60 of $\mathrm{H}_{2} \mathrm{O}: \mathrm{D}_{2} \mathrm{O}$ in the crystallization buffer. For the lowest protonation level explored (10\%), the average SNR is 18.9. A gradual increase in SNR is obtained up to $40 \%$ exchangeable protons with a SNR value of 44.6. The SNR then decreases to 11.1 for the sample which was recrystallized with $100 \% \mathrm{H}_{2} \mathrm{O}$. The SNR ratio for the $30 \%$ sample (41.1) is similar to the $40 \%$ sample, indicating a broad maximum for a proton density of $30-40 \%$ at exchangeable sites. The given SNR values discussed so far do not take into account the reduction of the recycle delay induced by a larger concentration of protons in the sample (Table 1). Considering the effective proton $T_{1}$ relaxation time, the SNR per unit time (SNRT) can be calculated (see Fig. 2a). Obviously, the same NMR experiment can be recorded faster for samples which contain more protons and which accordingly have a shorter ${ }^{1} \mathrm{H} T_{1}$ relaxation time. The SNRT is increased for samples which were recrystallized with more than $20 \% \mathrm{H}_{2} \mathrm{O}$ in the crystallization buffer. The maximum value is obtained for the $40 \%$ sample yielding a signal-to-noise value of $\sim 80.1$. The SNRT values for 10 selected residues are shown separately in Fig. 2b (for T24, K27, L33, N35, V44, A55, K59, K60, L61, and D62). It should be noted that the disappearing resonances have their maximum SNR already at $\sim 30 \%$ $\mathrm{H}_{2} \mathrm{O}$, whereas, the remaining resonances $(15$ out of 48 
resolved resonances) at $40 \% \mathrm{H}_{2} \mathrm{O}$. Moreover, there is no correlation of the proximity of a specific amide proton to side chain exchangeable protons which potentially might

(a)

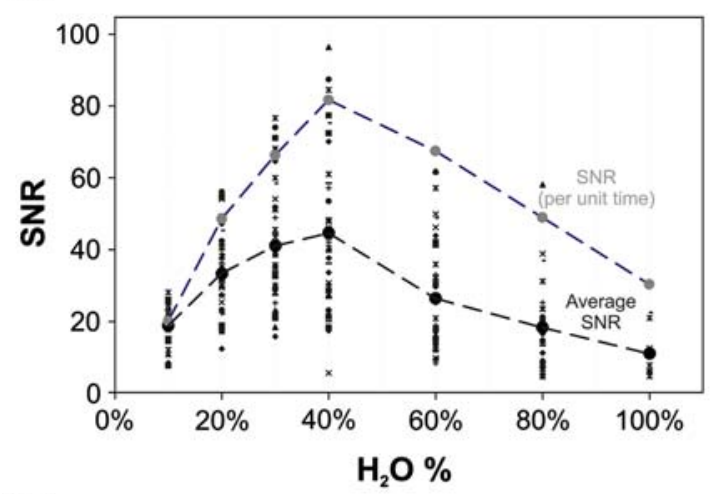

(b)

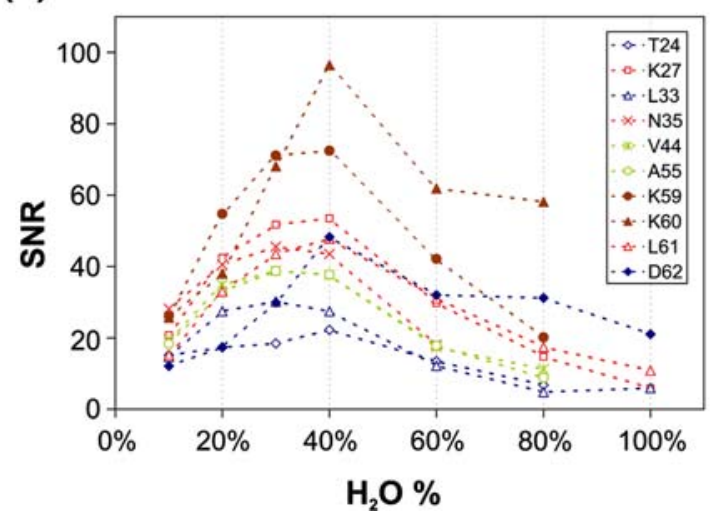

Fig. 2 a Average cross peak SNR obtained from $2 \mathrm{D}{ }^{1} \mathrm{H},{ }^{15} \mathrm{~N} \mathrm{CP}$ correlation spectra for perdeuterated $\alpha$-spectrin $\mathrm{SH} 3$ at different concentrations of $\mathrm{H}_{2} \mathrm{O}$ in the crystallization buffer. Residue specific SNRs are represented with symbols. The SNR and SNRT (signal-tonoise per unit time) is indicated in large black and grey symbols, respectively. The SNRT is calculated using the equation $\mathrm{S} / \mathrm{N}$ (per unit time) $=\mathrm{S} / \mathrm{N} \sqrt{\frac{7}{T_{1} \times 1.3}}$ where 7 corresponds to the recycle compromise proton resolution. Instead, variations in linewidths seem to be more or less randomly distributed. A higher protonation level may be desirable when a shorter (c)
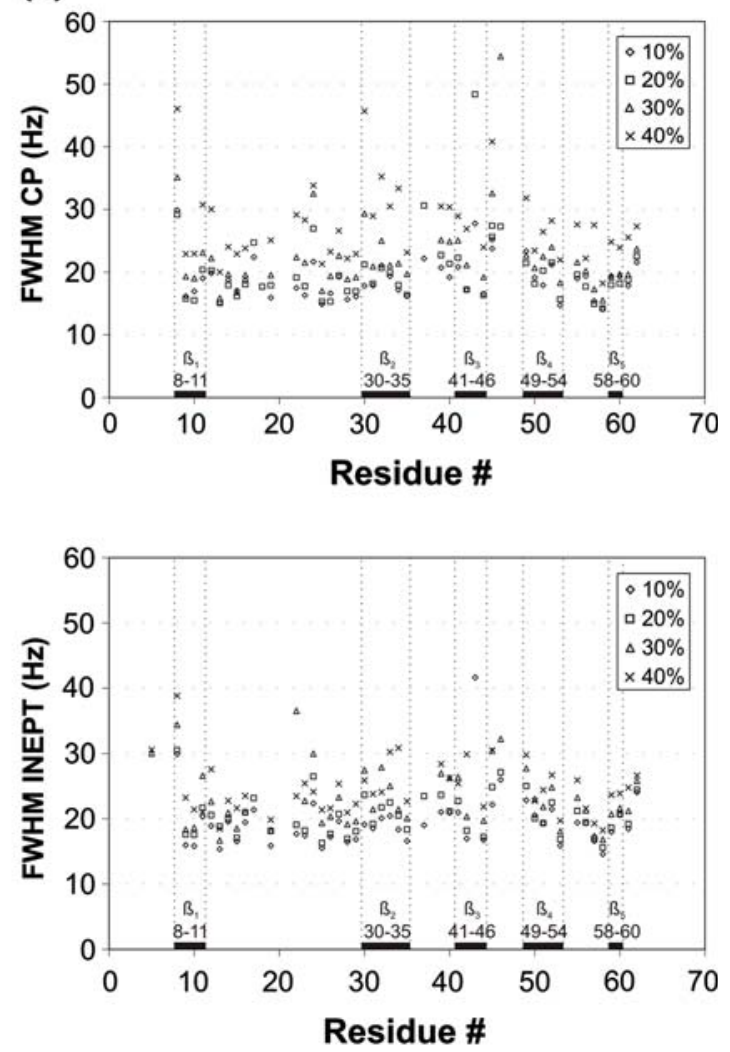

delay used in the NMR experiments. b SNR values for 10 selected amino acids as a function of the $\mathrm{H}_{2} \mathrm{O}$ concentration in the crystallization buffer. c Amide proton line widths of residues in the $\alpha$-spectrin SH3 domain at different $\mathrm{H}_{2} \mathrm{O}$ concentration in $\mathrm{CP}$ and INEPT based experiments. $\beta$-sheet regions in the $\mathrm{SH} 3$ domain are indicated along the primary sequence
Fig. $32 \mathrm{D}{ }^{1} \mathrm{H},{ }^{15} \mathrm{~N} \mathrm{CP}$ correlation spectra of the $\mathrm{SH} 3$ domain for samples recrystallized with 20,60 and $100 \% \mathrm{H}_{2} \mathrm{O}$ in the crystallization buffer $(275 \mathrm{~K}, 24 \mathrm{kHz}$ MAS).

The spectrum recorded with the sample prepared with a concentration of $20 \%$ exchangeable protons is shown in red. Two classes of cross peaks are highlighted belonging to residues which are either remaining or disappearing in the spectra of the sample prepared with $100 \% \mathrm{H}_{2} \mathrm{O}$ in the crystallization buffer
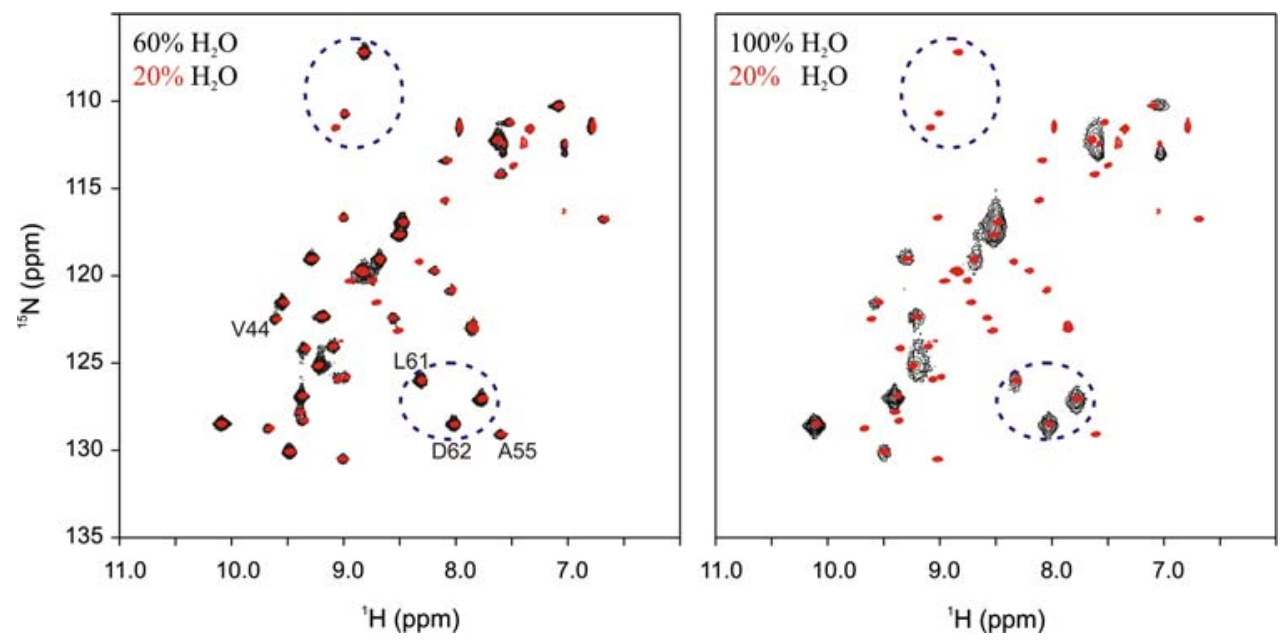
recycle delay is of greater advantage than the reduced linewidth. For example in 3D or 4D experiments, the full evolution time usually can not be digitized due to time constraints. Alternatively, reduced relaxation times can be achieved using Paramagnetic Relaxation Enhancement (PRE) (Linser et al. 2007; Wickramasinghe and Ishii 2007).

In Fig. 2c, the experimental linewidths obtained from $2 \mathrm{D}{ }^{1} \mathrm{H}-{ }^{15} \mathrm{~N} \mathrm{CP}$ and INEPT based correlation experiments are shown. An increase in proton density results in increased linewidths in both the ${ }^{1} \mathrm{H}$ and ${ }^{15} \mathrm{~N}$ dimensions (see Table 1). Interestingly, there is a larger variation of proton linewidth in $\mathrm{CP}$ based experiments at high concentrations of $\mathrm{H}_{2} \mathrm{O}$ compared to experiments where INEPT transfers were employed. However, we did not find a discernable trend according to residue type and mobility.

The proton-detected $2 \mathrm{D}{ }^{1} \mathrm{H}-{ }^{15} \mathrm{~N}$ CPMAS NMR spectra of the SH3 samples with 60 and 100\% exchangeable protons are shown in Fig. 3a, b respectively. For comparison, the spectra are superimposed with a spectrum recorded for the $20 \%$ sample (red). The average proton linewidths for the samples containing 20,60, and $100 \%$ exchangeable protons are 20.2, 32.5, and $58.3 \mathrm{~Hz}$, respectively. From Fig. 3a, it is apparent that many resonances are less intense for the $60 \%$ sample compared to $20 \%$ sample. However, still all resonances can be observed. As the proton density is increased, the resonances broaden further. Several correlations can only be identified based on previous assignments. We separated amide correlations into two groups, depending on whether they remain or disappear in the ${ }^{1} \mathrm{H}$, ${ }^{15} \mathrm{~N}$ correlation spectra, as highlighted in Fig. 3. At the highest concentration of $\mathrm{H}_{2} \mathrm{O}(100 \%)$, more than $60 \%$ of the resonances have broadened beyond detection, while the ones that remain have average proton and nitrogen linewidths on the order of 58.3 and $41.2 \mathrm{~Hz}$, respectively.

An analysis of the amide proton peak shapes as a function of the amount of $\mathrm{H}_{2} \mathrm{O}$ in the crystallization buffer is shown in Fig. 4a, b for four selected residues. These residues are found to be mobile and rigid, respectively, according to their ${ }^{15} \mathrm{~N}$ relaxation rates reported previously (Chevelkov et al. 2008; Chevelkov and Reif 2008). V44 and A55 are located in regular secondary structure elements $\left(\beta\right.$-sheets $\left.\beta_{3} / \beta_{4}\right)$ and disappear in spectra of samples which were prepared with more than $60 \% \mathrm{H}_{2} \mathrm{O}$ in the crystallization buffer. For these residues, the MAS rate (Zhou and Rienstra 2008) and internal dynamics (Chevelkov et al. 2008) is apparently insufficient to average the homonuclear dipolar coupling network. Two residues that are not as severely broadened, L61 and D62 are located in the C-terminus of the SH3 domain. These two residues (a)

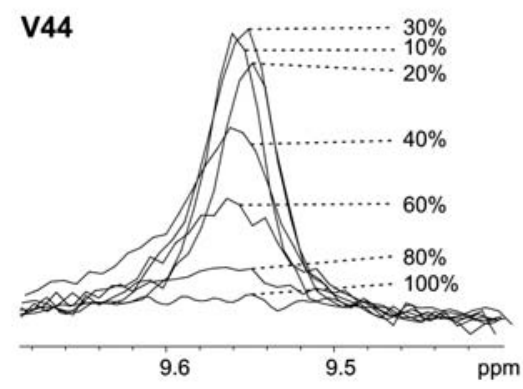

(b)

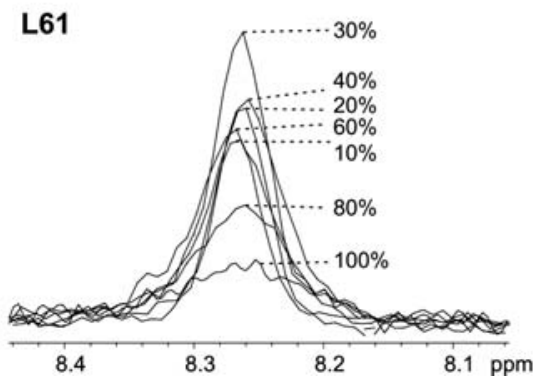

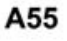

A55

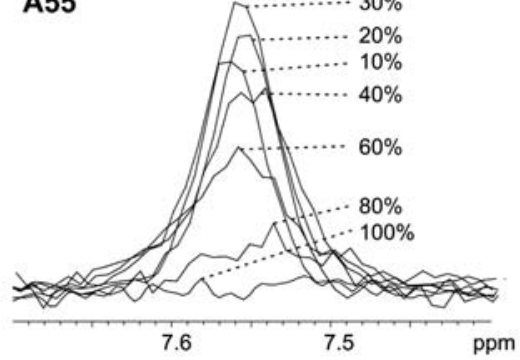

D62

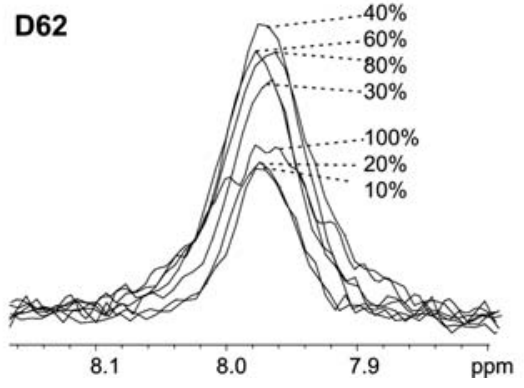

(c)

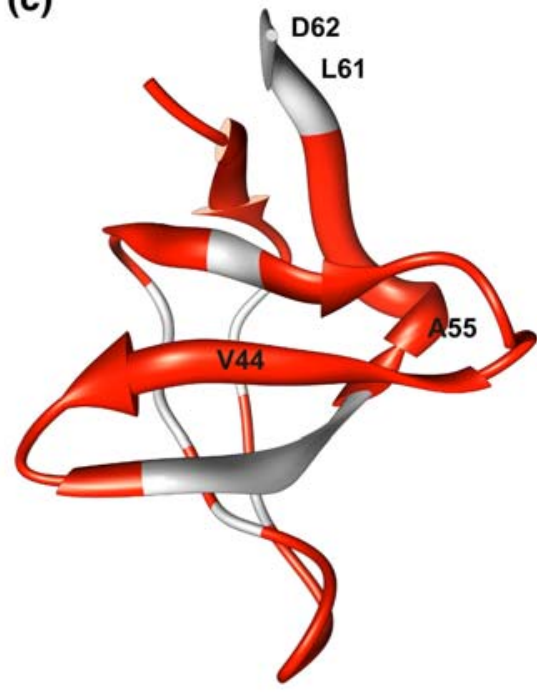

Fig. $4{ }^{1} \mathrm{H}^{\mathrm{N}}$ traces extracted from selected resonances in ${ }^{1} \mathrm{H},{ }^{15} \mathrm{~N}$ correlation spectra recorded for different concentration of $\mathrm{H}_{2} \mathrm{O}$. Residues which are disappearing (V44, A55) and which are still observable (L61, D62) at a proton density of $100 \% \mathrm{H}_{2} \mathrm{O}$ are represented in (a) and (b), respectively. (c) Schematic representation of the structure of the SH3 domain, highlighting residues for which
${ }^{1} \mathrm{H},{ }^{15} \mathrm{~N}$ correlation signals remain visible in the sample that we recrystallized with $100 \% \mathrm{H}_{2} \mathrm{O}$ (grey). Resonances of residues which disappear are color coded in red. At very high proton concentration, in particular resonances of residues located in mobile regions are visible, whereas resonances of residues in rigid parts of the protein tend to disappear 
have increased dynamics, which facilitates the averaging of the dipolar coupling, and therefore yield more narrow resonance lines. A structural representation of the two types of amide moieties is shown in Fig. 4c. The 16 amino acids for which we still find reasonable cross peak shapes in the sample prepared with $100 \% \mathrm{H}_{2} \mathrm{O}$ (grey) are located in mobile regions. Resonances that disappear (red) are generally stemming from residues located in the more rigid beta-sheet regions. For L8 and D62 (Fig. 4c), which have been shown to be the most mobile residues of the $\mathrm{SH} 3$ domain (Chevelkov and Reif 2008), the signals remain even in the sample crystallized with $100 \% \mathrm{H}_{2} \mathrm{O}$. The observation that mobile residues remain detectable at the highest levels of protonation supports the hypothesis that internal dynamics facilitate averaging of ${ }^{1} \mathrm{H},{ }^{1} \mathrm{H}$ homonuclear dipolar couplings.

A quantitative characterization of the differences between dipolar and scalar coupling mediated transfer mechanisms shows that the INEPT scheme produces larger cross peak intensities compared to $\mathrm{CP}$ for the $10 \%$ protonated SH3 sample (Chevelkov and Reif 2008). We have extended this study by performing CP and INEPT type experiments for samples in which we varied the amount of $\mathrm{H}_{2} \mathrm{O}$ employed for crystallization from 10 up to $40 \%$. The SNR obtained for CP experiments reaches a maximum value of 44.6 at $40 \%$ exchangeable protons. On the other hand, the SNR for INEPT experiments remains nearly the same from 10 up to $30 \%(\mathrm{SNR}=\sim 50)$, which is larger than the maximum SNR obtained for $\mathrm{CP}$ experiments. Figure 5 shows the ratio of intensities obtained in $\mathrm{CP}$ and INEPT type experiments as a function of the amount of $\mathrm{H}_{2} \mathrm{O}$ in the crystallization buffer. Upon increase of the proton density, CP based experiments gain over experiments which use INEPT for magnetization transfer. However, up to ca. $35 \% \mathrm{H}_{2} \mathrm{O}$, INEPT experiments have overall

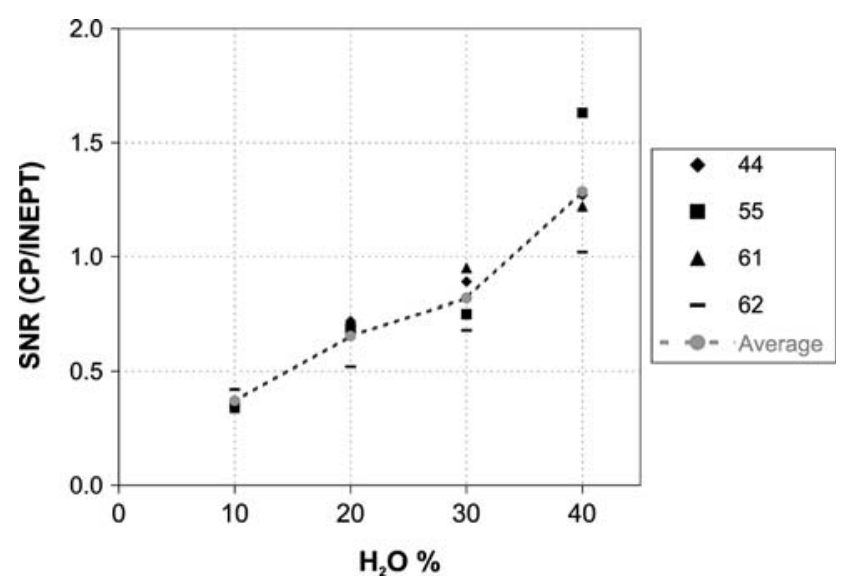

Fig. 5 Relative signal-to-noise ratios (CP versus INEPT) for four selected residues of the $\mathrm{SH} 3$ domain as a function of the amount of $\mathrm{H}_{2} \mathrm{O}$ in the sample a higher SNR. On the other hand, water suppression turned out to be more difficult in INEPT compared to CP experiments. This might change when pulsed field gradients become routinely available for solid-state NMR applications.

All the experiments presented in this work have been performed at $24 \mathrm{kHz}$ of MAS spinning frequency. For the samples we have studied, this spinning frequency was sufficient for obtaining high resolution spectra. Though, it might be beneficial for the higher protonation levels to spin at higher frequencies to suppress the residual dipolar coupling more efficiently to remove the effects, as visible in Fig. 3. For example, in a recent work of Reif et. al, the effect of MAS frequency to the observed proton linewidths was investigated for a similar system (Chevelkov et al. 2006). It was shown that even at $10 \% \mathrm{H}_{2} \mathrm{O}$, the proton dipolar couplings are not totally suppressed. As a result, higher spinning frequency for high protonation might still be beneficial in this respect to reduce residual dipolar coupling. According to our linewidth fitting, the extrapolated proton linewidth was determined to be $\sim 16 \mathrm{~Hz}$ at either very high MAS frequency or at very low $\mathrm{H}_{2} \mathrm{O} \%$ (not shown here).

\section{Conclusion}

We have presented a detailed analysis of the effect of the concentration of exchangeable protons in perdeuterated proteins on resolution and sensitivity in ${ }^{1} \mathrm{H},{ }^{15} \mathrm{~N}$ correlation spectra. We observe a threefold increase in linewidth when the concentration of $\mathrm{H}_{2} \mathrm{O}$ in the crystallization buffer is raised from 10 to $100 \%$, yielding average ${ }^{1} \mathrm{H}$ linewidths from 19 to $58 \mathrm{~Hz}$, respectively. We suggest a concentration of $30-40 \%$ exchangeable protons as the best compromise between sensitivity and resolution at a MAS frequency of $24 \mathrm{kHz}$, yielding the highest SNR in $\mathrm{CP}$ and INEPT based experiments. 3.8 times more signal is obtained from $40 \% \mathrm{H}_{2} \mathrm{O}$ sample compared to $10 \%$ in the CP based experiment, whereas the proton linewidth is only 1.5 times worse. Above $40 \%$, spectral resolution and sensitivity deteriorates and CP based experiments become superior to experiments using INEPT type transfers. Under these experimental conditions, amides of rigid residues appear to be more broadened compared to amides located in mobile regions which seem not to be as severely affected. We believe that the optimization of the concentration of exchangeable protons in perdeuterated proteins is crucial for biological systems in which sensitivity is an issue. Therefore, the increased sensitivity we find for proton detected experiments in the solid-state, will increase the popularity of the use of perdeuterated proteins even further. 


\section{References}

Chevelkov V, Reif B (2008) TROSY effects in MAS solid-state NMR. Concepts Magn Reson Part B 32:143-156

Chevelkov V, Rossum BJ, Castellani F, Rehbein K, Diehl A, Hohwy M, Steuernagel S, Engelke F, Oschkinat H, Reif B (2003) ${ }^{1} \mathrm{H}$ detection in MAS solid-state NMR spectroscopy of biomacromolecules employing pulsed field gradients for residual solvent suppression. J Am Chem Soc 125:7788-7789

Chevelkov V, Rehbein K, Diehl A, Reif B (2006) Ultrahigh resolution in proton solid-state NMR spectroscopy at high levels of deuteration. Angew Chem Int Ed 45:3878-3881

Chevelkov V, Diehl A, Reif B (2008) Measurement of ${ }^{15} \mathrm{~N}-\mathrm{T}_{1}$ relaxation rates in a perdeuterated protein by magic angle spinning solid-state nuclear magnetic resonance spectroscopy. J Chem Phys 128:052316

Ishii Y, Tycko R (2000) Sensitivity enhancement in solid state ${ }^{15} \mathrm{~N}$ NMR by indirect detection with high-speed magic angle spinning. J Magn Reson 142:199-204

Ishii Y, Yesinowski JP, Tycko R (2001) Sensitivity enhancement in solid-state ${ }^{13} \mathrm{C}$ NMR of synthetic polymers and biopolymers by ${ }^{1} \mathrm{H}$ NMR detection with high-speed magic angle spinning. J Am Chem Soc 123:2921-2922

Laage S, Sachleben JR, Steuernagel S, Pierattelli R, Pintacuda G, Emsley L (2009) Fast acquisition of multi-dimensional spectra in solid-state NMR enabled by ultra-fast MAS. J Magn Reson 196:133-141

Leskes M, Madhu PK, Vega S (2007) A broad-band z-rotation windowed phase-modulated Lee-Goldburg pulse sequence for ${ }^{1} \mathrm{H}$ spectroscopy in solid-state NMR. Chem Phys Lett 447:370-374

Linser RJ, Chevelkov V, Diehl A, Reif B (2007) Sensitivity enhancement using paramagnetic relaxation in MAS solid-state NMR of perdeuterated proteins. J Magn Reson 189:209-216

Linser R, Fink U, Reif B (2008) Proton-detected scalar coupling based assignment strategies in MAS solid-state NMR spectroscopy applied to perdeuterated proteins. J Magn Reson 193:89-93
Morcombe CR, Paulson EK, Gaponenko V, Byrd A, Zilm KW (2005) ${ }^{1} \mathrm{H}-{ }^{5} \mathrm{~N}$ correlation spectroscopy of nanocrystalline proteins. J Bio NMR 31:217-230

Paulson EK, Morcombe CR, Gaponenko V, Dancheck B, Byrd RA, Zilm KW (2003) Sensitive high resolution inverse detection NMR spectroscopy of proteins in the solid state. J Am Chem Soc 125:15831-15836

Rossum BJ, Castellani F, Pauli J, Rehbein K, Hollander J, de Groot HJM, Oschkinat H (2003) Assignment of amide proton signals by combined evaluation of $\mathrm{HN}, \mathrm{NN}$ and HNCA MAS-NMR correlation spectra. J Biomol NMR 25:217-223

Salager E, Stein RS, Steurnagel S, Lesage A, Elena B, Emsley L (2009) Enhanced sensitivity in high-resolution ${ }^{1} \mathrm{H}$ solid-state NMR spectroscopy with DUMBO dipolar decoupling under ultra-fast MAS. Chem Phys Lett 469:336-341

Vinogradov E, Madhu PK, Vega S (1999) High-resolution proton solid-state NMR spectroscopy by phase-modulated Lee-Goldburg experiment. Chem Phys Lett 314:443-450

Wickramasinghe NP, Ishii Y (2007) Sensitivity enhancement in ${ }^{13} \mathrm{C}$ solid-state NMR of protein microcrystals by use of paramagnetic metal ion for optimizing ${ }^{1} \mathrm{H}^{\mathrm{T}} \mathrm{T}_{1}$ relaxation. J Magn Reson 184:350-356

Zhou DH, Rienstra CM (2008) Rapid analysis of organic compounds by proton-detected heteronuclear correlation NMR spectroscopy with $40 \mathrm{kHz}$ magic-angle spinning. Angew Chem 47:7328-7331

Zhou DH, Graesser DT, Franks WT, Rienstra CM (2006) Sensitivity and resolution in proton solid-state NMR at intermediate deuteration levels: quantitative linewidth characterization and applications to correlation spectroscopy. J Magn Reson 178: 297-307

Zhou DH, Shah G, Cormos M, Mullen C, Sandoz D, Rienstra CM (2007) Proton-detected solid-state NMR spectroscopy of fully protonated proteins at $40 \mathrm{kHz}$ magic-angle spinning. J Am Chem Soc 129:11791-11801 